\title{
Transient forebrain ischemia under hyperthermic condition accelerates memory impairment and neuronal death in the gerbil hippocampus by increasing NMDAR1 expression
}

\author{
BORA KIM $^{1^{*}}$, JI HYEON AHN ${ }^{1,2^{*}}$, DAE WON KIM ${ }^{3}$, TAE-KYEONG LEE ${ }^{4}$, \\ YOON SUNG KIM ${ }^{5,6}$, MYOUNG CHEOL SHIN ${ }^{5}$, JUN HWI CHO ${ }^{5}$, YOUNG-MYEONG KIM ${ }^{7}$, \\ JOON HA PARK ${ }^{8}$, IL JUN KANG ${ }^{9}$, JAE-CHUL LEE $^{1}$ and MOO-HO WON ${ }^{1}$
}

\begin{abstract}
${ }^{1}$ Department of Neurobiology, School of Medicine, Kangwon National University, Chuncheon, Gangwon 24341;
${ }^{2}$ Department of Physical Therapy, College of Health Science, Youngsan University, Yangsan, Gyeongnam 50510;

${ }^{3}$ Department of Biochemistry and Molecular Biology, and Research Institute of Oral Sciences, College of Dentistry, Gangnung-Wonju National University, Gangneung, Gangwon 25457; ${ }^{4}$ Department of Biomedical Science, Research Institute of Bioscience and Biotechnology, Hallym University, Chuncheon, Gangwon 24252; ${ }^{5}$ Department of Emergency Medicine, Kangwon National University Hospital, School of Medicine, Kangwon National University, Chuncheon, Gangwon 24289;

${ }^{6}$ Department of Emergency Medicine, Samcheok Medical Center, Samcheok, Kangwon 25920; ${ }^{7}$ Department of Molecular and Cellular Biochemistry, School of Medicine, Kangwon National University, Chuncheon, Gangwon 24341;

${ }^{8}$ Department of Anatomy, College of Korean Medicine, Dongguk University, Gyeongju, Gyeongbuk 38066;

${ }^{9}$ Department of Food Science and Nutrition, Hallym University, Chuncheon, Gangwon 24252, Republic of Korea
\end{abstract}

Received August 11, 2020; Accepted November 30, 2020

DOI: $10.3892 / \mathrm{mmr} .2021 .11895$

\begin{abstract}
Altered expression levels of $N$-methyl-D-aspartate receptor (NMDAR), a ligand-gated ion channel, have a harmful effect on cellular survival. Hyperthermia is a proven risk factor of transient forebrain ischemia (tFI) and can cause extensive and severe brain damage associated with mortality. The objective of the present study was to investigate whether hyperthermic preconditioning affected NMDAR1 immunoreactivity associated with deterioration of neuronal function in the gerbil hippocampal CA1 region following tFI via histological and western blot analyses. Hyperthermic preconditioning was performed for $1 \mathrm{~h}$ before tFI, which was developed by ligating common carotid arteries for $5 \mathrm{~min}$. tFI-induced cognitive impairment under hyperthermia was worse compared with that under normothermia. Loss (death) of pyramidal neurons in the CA1 region occurred fast and was more severe
\end{abstract}

Correspondence to: Professor Moo-Ho Won or Dr Jae-Chul Lee, Department of Neurobiology, School of Medicine, Kangwon National University, 1 Kangwondaehak-gil, Chuncheon, Gangwon 24341, Republic of Korea

E-mail:mhwon@kangwon.ac.kr

E-mail: anajclee@kangwon.ac.kr

${ }^{*}$ Contributed equally

Key words: cognition, hyperthermic preconditioning, ligand-gated ion channel, neuronal death, pyramidal neurons, transient ischemia under hyperthermia compared with that under normothermia. NMDAR1 immunoreactivity was not observed in the somata of pyramidal neurons of sham gerbils with normothermia. However, its immunoreactivity was strong in the somata and processes at $12 \mathrm{~h}$ post-tFI. Thereafter, NMDAR1 immunoreactivity decreased with time after tFI. On the other hand, NMDAR1 immunoreactivity under hyperthermia was significantly increased in the somata and processes at $6 \mathrm{~h}$ post-tFI. The change pattern of NMDAR1 immunoreactivity under hyperthermia was different from that under normothermia. Overall, accelerated tFI-induced neuronal death under hyperthermia may be closely associated with altered NMDAR1 expression compared with that under normothermia.

\section{Introduction}

Transient forebrain ischemia (tFI) can be induced by temporary deprivation of arterial blood flow to the forebrain through occlusion of bilateral common carotid arteries for five minutes. A tFI can cause death (loss) of pyramidal cells (neurons) only in the Conus Ammonis 1 region (CA1) of the hippocampus of the gerbil (1). Pyramidal cells of the CA1 are called CA1 pyramidal cells. They will die at 4-5 days after tFI for $5 \mathrm{~min}$. This phenomenon of CA1 pyramidal cell death is called delayed neuronal death (DND) (1). Afterward, DND has been reported in the hippocampal CA1 of rodents including rats and mice $(2,3)$.

Possible mechanisms of DND after tFI have been reported. Among them, transient increase in excitatory amino acid transmitter efflux is probably associated with the degrees of neuronal damage or death in ischemic brains (4). In detail, 
during ischemia, high extracellular glutamate level can activate ionotropic glutamate receptors (i.e., $N$-methyl-D-aspartate receptor (NMDAR) that are ligand-gated ion channels leading to channel opening and overflow of calcium ions into neurons. Finally, neuronal damage/death occurs $(5,6)$. Many researchers have reported that brain diseases and dysfunctions are related to abnormal NMDAR expressions $(7,8)$. For example, it has been reported that pathological activation of NMDAR1 is a major cause of neuronal death following brain ischemia (9-12).

Brain or body temperature has been thought to be one of major factors affecting neuronal survival or death after brain ischemic injury (13-15). Preclinical studies have provided evidence that hyperthermia has harmful effects on animal models of transient global cerebral ischemia $(16,17)$ and transient focal cerebral ischemia models $(18,19)$. Clinical reports have confirmed that hyperthermia can accelerate infarction and worsen outcomes of ischemic stroke patients $(20,21)$. Recently, we have reported that hyperthermia can accelerate and worsen neuronal damage or death in the gerbil hippocampus after tFI $(13,22,23)$.

As described above, transient ischemic insults under hyperthermic condition can lead to extensive brain damage. However, molecular mechanisms involved in deleterious effects of hyperthermic condition on results from tFI have not yet been fully clarified. Thus, the objective of this study was to investigate changes of NMDAR1 expressions in the hippocampus and relationships between NMDAR 1 changes and neuronal damage or death after tFI induction under hypothermic condition in gerbils.

\section{Materials and methods}

Experimental animals, protocol and groups. Male Mongolian gerbils (age, 6 months; body weight, 64-74 g) were used. They were obtained from the Experimental Animal Center located in Kangwon National University (Chuncheon, Kangwon, Korea). The protocol of this research was approved (approval number, KW-200113-1) on January 13, 2020 by the Institutional Animal Care and Use Committee (IACUC) located in Kangwon National University. The gerbils used in this study were cared under constant temperature $\left(\sim 23^{\circ} \mathrm{C}\right)$ and humidity $(\sim 55 \%)$ with a 12-h light/dark cycle. The handling and caring animals conformed to the guidelines in the 'Current international laws and policies' of the 'NIH Guide for the Care and Use of Laboratory Animals' (The National Academies Press, 8th edition, 2011). Number of the gerbils used for this study were minimized, and the suffering caused by our procedures used in this experiment was minimized.

Experimental animals (total number $=120$ ) were assigned to four groups: i) sham-operated animals with normothermia (NT) (NT/sham group, $n=12)$; ii) tFI-operated animals with NT (NT/tFI group, $\mathrm{n}=12)$; iii) sham-operated animals with hyperthermia (HT) (HT/sham group, $n=48)$; and iv) tFI-operated animals with HT (HT/tFI group, $n=48)$.

Induction of $t F I$. As previously described (23), the gerbils to be used for tFI were anesthetized with mixture of $2.5 \%$ isoflurane in $33 \%$ oxygen and $67 \%$ nitrous oxide via inhalation. Under anesthesia, hyperthermia was induced by heating them with a heating pad until rectal temperature reached $39.5 \pm 0.2^{\circ} \mathrm{C}$ for $1 \mathrm{~h}$ before $\mathrm{tFI}$ induction. For normothermia, rectal temperature was regulated at $37 \pm 0.2^{\circ} \mathrm{C}$. $\mathrm{tFI}$ was induced as follows. Both common carotid arteries were isolated from the carotid sheath and occluded using aneurysm clips for $5 \mathrm{~min}$. Thereafter, the gerbils were kept in thermal incubator (temperature, $23^{\circ} \mathrm{C}$; humidity, $60 \%$ ) to recover to normothermic level. The gerbils of NT/sham and HT/sham groups received the same surgical process of tFI without bilateral carotid artery occlusion.

Passive avoidance test (PAT). Short term memory was evaluated by PAT used by previous researchers $(24,25)$ with modifications. In short, we used a Gemini Avoidance System from GEM 392 (San Diego Instruments) consisting of two (light and dark) compartments which communicates through a sliding door was used. The gerbils received training of PAT for three days before tFI. Namely, the gerbils were allowed to explore light and dark compartments for $1 \mathrm{~min}$ while the sliding door was opened. And, when the gerbils enter the dark compartment, the door was closed, and they received an electric foot-shock $(0.5 \mathrm{~mA}$ for $5 \mathrm{sec})$ from a steel grid in the floor. Two and five days after tFI, PAT was performed. The PAT was elevated as latency time. Namely, the gerbils were put in the light compartment, while the door was opened. The latency time was that the gerbils in the light compartment went into the dark compartment after receiving the electric foot-shock. Maximum latency time was $180 \mathrm{sec}$, which was to stay in the light compartment with the an electric foot-shock.

Western blot analysis. The gerbils ( $\mathrm{n}=7$ at $6 \mathrm{~h}, 1$ day, 2 days, and 5 days after tFI) in each group were deeply anesthetized by intraperitoneal injection of $60 \mathrm{mg} / \mathrm{kg}$ pentobarbital sodium and sacrificed to analyze the level of NMDAR1 protein in the hippocampal CA1 region after tFI. As previously described (26), the hippocampi were removed, and the CA1 regions were obtained. The tissues of the CA1 regions were homogenized in PBS (5 mM, pH 7.4), which contained $0.2 \%$ Nonidet P-40, $0.1 \mathrm{mM}$ ethylene glycol bis (2-aminoethyl Ether)-N,N,N',N' tetraacetic acid (EGTA) (pH 8.0), $10 \mathrm{mM}$ ethylendiamine tetraacetic acid (EDTA) (pH 8.0), $15 \mathrm{mM}$ sodium pyrophosphate, $150 \mathrm{mM} \mathrm{NaCl}$, $50 \mathrm{mM} \mathrm{NaF}, 2 \mathrm{mM}$ sodium orthovanadate, $100 \mathrm{mM}$ $\beta$-glycerophosphate, $1 \mathrm{mM}$ dithiothreitol (DTT) and $1 \mathrm{mM}$ phenylmethylsulfonyl fluoride (PMSF). The homogenates were separated by centrifugation, and the protein level was determined in the supernatants with Micro BCA protein assay kit (Pierce Chemical Co.). Aliquots containing total protein $(20 \mu \mathrm{g})$ were boiled in loading buffer $(150 \mathrm{mM}$ Tris, pH 6.8) containing 6\% SDS, $0.3 \%$ bromophenol blue, $3 \mathrm{mM}$ DTT, and $30 \%$ glycerol and loaded onto $12.5 \%$ polyacrylamide gel. Subsequently, they were received electrophoresis, and the gels were transferred to nitrocellulose transfer membranes. To block non-specific staining, the membranes were incubated with $5 \%$ defatted milk at room temperature for $60 \mathrm{~min}$. The membranes, thereafter, were immunoreacted with rabbit anti-NMDAR1 (diluted 1:1,000, Abcam), peroxidase-conjugated goat anti-rabbit IgG (diluted 1:250, Sigma-Aldrich Co.; Merck KGaA) and ECL kit (Pierce Chemical Co.). 
The analysis of western blotting was done according to a published method (26). In brief, the western bands were scanned with computer scanner, and the quantification of the analysis was done using a Scion Image software from Scion Corp. The expression rate of NMDAR1 protein was normalized through the corresponding expression rate of $\beta$-actin.

Tissue processing for histology. Sections containing the hippocampus were prepared as previously described (13). In short, the gerbils in each group were deeply anesthetized by intraperitoneal injection of $60 \mathrm{mg} / \mathrm{kg}$ pentobarbital sodium at $6 \mathrm{~h}, 1$ day, 2 days, and 5 days after tFI. Under anesthesia, the brains of the gerbils were perfused with $4 \%$ paraformaldehyde solution (in 0.1 M PB, $\mathrm{pH}$ 7.4). The brains were removed and more fixed in the fixative for $4 \mathrm{~h}$. The brain tissues were infiltrated with solution of $30 \%$ sucrose to avoided tissue damage from freeze. The brain tissues were frontally sectioned into $30-\mu \mathrm{m}$ sections in a cryostat, and they were stored in well plates containing PBS ( $\mathrm{pH} 7.4)$.

Histofluorescence with Fluoro-Jade B. Histofluorescence with Fluoro-Jade B (F-JB, a fluorescent marker of cellular degeneration or death) was carried out to examine damage/death of cells or neurons in gerbil hippocampus after tFI. In short, as described in our published papers $(13,23)$. the prepared brain sections were immersed in solution of $0.06 \%$ potassium permanganate and stained with solution of $0.0004 \%$ F-JB (Histochem).

Eight sections with $90-\mu \mathrm{m}$ interval were selected in reference to anatomical landmarks of the gerbil brain atlas (27). The samples were observed using a fluorescence microscope from Carl Zeiss with blue excitation fluorescence filter between 450-490 nm. Images of F-JB positive cells, which underwent degeneration, as bright fluoresce, when compared to the background (28), were captured and counted with NIH ImageJ 1.59 software.

Immunohistochemistry. Immunohistochemistry was performed to examine neurons using rabbit anti-neuronal nuclei (NeuN) (diluted 1:1,100, Chemicon International) and NMDAR1 using rabbit anti-NMDAR1 (diluted 1:100, Abcam) according to method in our published papers $(13,23)$. Briefly, the prepared sections were treated with solution of $0.3 \%$ hydrogen peroxide $\left(\mathrm{H}_{2} \mathrm{O}_{2}\right)$ and followed by solution of $10 \%$ normal goat serum. Subsequently, these sections were incubated in each solution of primary antibody for $12 \mathrm{~h}$ at $4^{\circ} \mathrm{C}$. Thereafter these sections were incubated in biotinylated goat anti-rabbit IgG (diluted 1:250, Vector) and streptavidin peroxidase complex (diluted 1:250, Vector). Finally, these immunoreacted sections were visualized by soaking them in solution of 3,3'-diaminobenzidine tetrahydrochloride.

For the analysis of NeuN-positive neurons, images of NeuN-positive structures were taken from the CA1 with AxioM1 light microscope from Carl Zeiss. For numbers of NeuN-positive neuron, the images of NeuN-positive neurons were captured in a $200 \times 200 \mu \mathrm{m}^{2}$ at approximate middle of the CA1 region. The cell count was done by averaging the total cell numbers from all of the observed sections in each group.

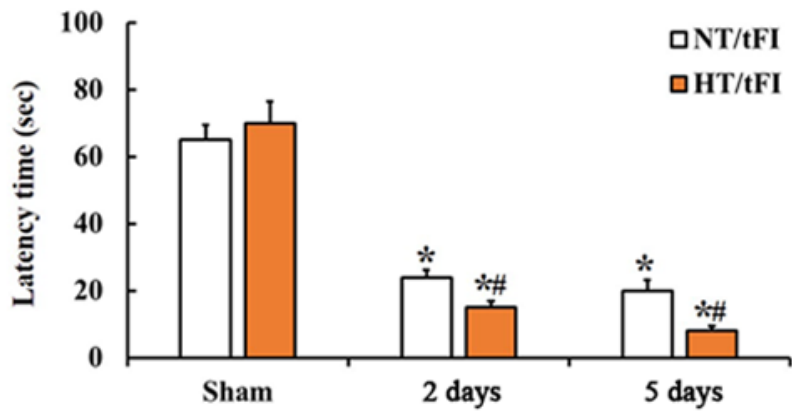

Figure 1. Passive avoidance test in the NT/sham, HT/sham, NT/tFI and HT/tFI groups at 2 and 5 days after tFI. At 2 and 5 days after tFI, the latency time in the $\mathrm{HT} / \mathrm{tFI}$ groups was significantly shorter than that in the respective NT/tFI group. The data are presented as the mean $\pm \mathrm{SEM}(\mathrm{n}=7)$. $\mathrm{P}<0.05$ vs. respective sham group; ${ }^{\#} \mathrm{P}<0.05$ vs. respective $\mathrm{NT} / \mathrm{tFI}$ group. $\mathrm{NT}$, normothermia; $\mathrm{HT}$, hyperthermia; tFI, transient forebrain ischemia.

The analysis of NMDAR1-immunoreactive structures in the area of interest (strata pyramidale of hippocampal CA1 region; Fig. S1) was evaluated as relative optical density (ROD), as \%. Images of NMDAR1-immunoreactive structures were capture like above-mentioned method. The background in the image was controlled, and ROD was calibrated with Adobe Photoshop version 8.0 and NIH Image 1.59 software according to method by (29).

Statistical analysis. Data presented here are expressed as the mean \pm SEM. The differences of the means between the groups were statistically analyzed. Analysis of variance (ANOVA) with a post hoc Bonferroni's multiple comparison tests with SPSS program was used to elucidate tFI-related differences among the experimental groups. In addition, two-way ANOVA was applied with the Bonferroni post hoc for comparison of two independent variables between the groups of normothermia and hyperthermia as well as their interaction. $\mathrm{P}<0.05$ was used for statistical significance.

\section{Results}

$P A T$. No significant difference in the latency time was shown between the NT/sham and HT/sham groups (Fig. 1). At 2 days after tFI, the latency time was significantly decreased in both $\mathrm{NT} / \mathrm{tFI}$ and HT/tFI groups, but, in the HT/tFI group, the latency time was significantly shorter (about $62.5 \%$ of the NT/tFI group) than that in the NT/tFI group (Fig. 1). At 5 days after tFI, the latency time in both of the groups was much shorter than that at 2 days after $\mathrm{tFI}$, showing that the latency time in the HT/tFI group was significantly shorter (about $40 \%$ of the NT/tFI group) than that in the NT/tFI group (Fig. 1).

\section{Neuronal damage/death}

NeuN-immunoreactive neurons. NeuN-immunoreactive neurons or cells in the NT/sham and HT/sham groups were easily shown in the stratum pyramidale (SP) consisted of pyramidal neurons in the $\mathrm{CA} 1$ region (Fig. 2Aa, $\mathrm{Ad}, \mathrm{Ba}$, and $\mathrm{Bd}$ ). In the NT/tFI group, NeuN-positive cells in the SP was rarely observed 5 days after tFI (Fig. 2Ac and Bc). However, numbers of NeuN-positive cells in the SP of the HT/tFI group were 


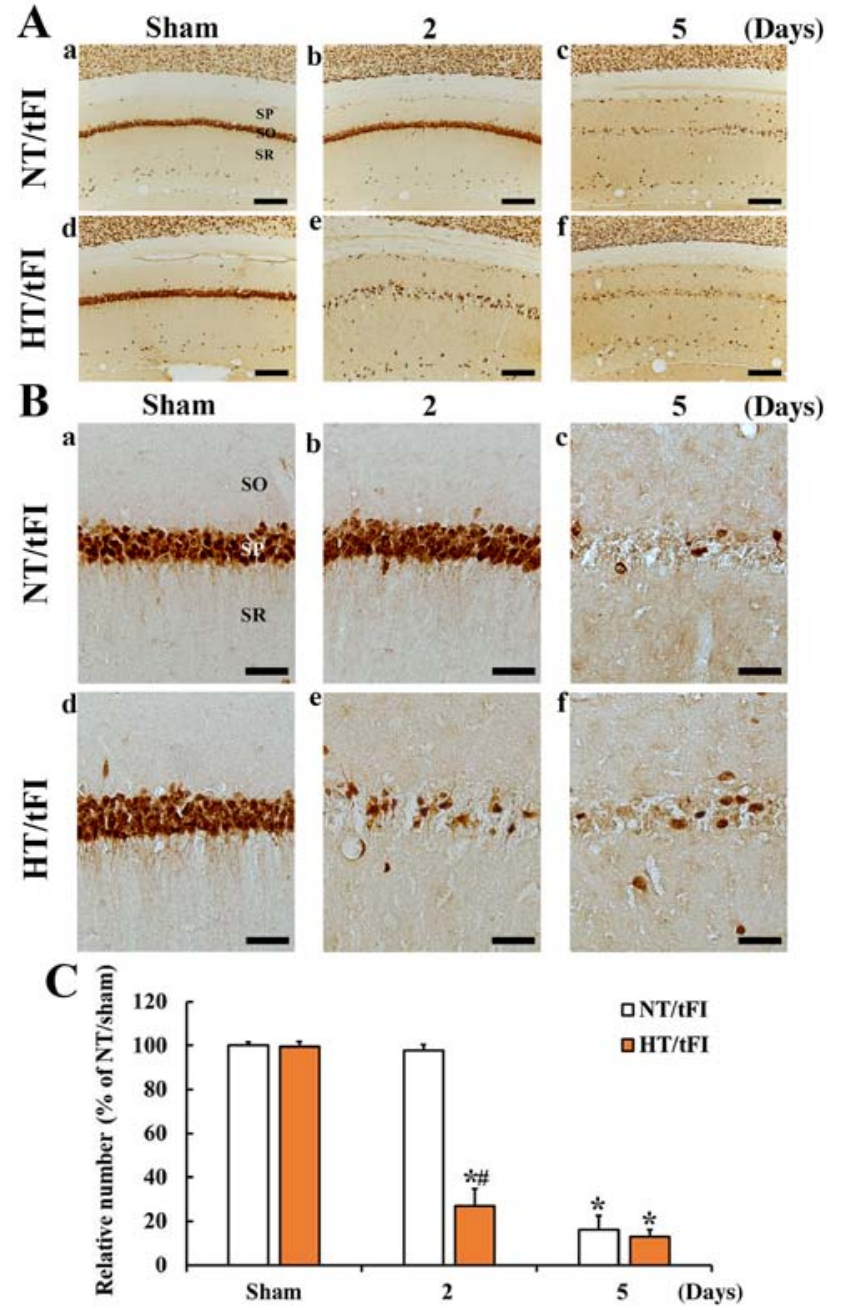

Figure 2. (A) Low (x10; scale bar, $200 \mu \mathrm{m}$ ) and (B) high (x40; scale bar, $50 \mu \mathrm{m}$ ) magnification of immunohistochemical staining for NeuN in the CA1 region of the (a) NT/sham, (b and c) NT/tFI at 2 and 5 days after tFI, (d) HT/sham and (e and f) HT/tFI groups at 2 and 5 days after tFI. Five days after $\mathrm{tFI}, \mathrm{NeuN}^{+}$neurons were rare in the SP. However, in the HT/tFI group, $\mathrm{NeuN}^{+}$neurons in the SP were dramatically decreased from 2 days after tFI. (C) Numbers of $\mathrm{NeuN}^{+}$neurons in the CA1 region. The data are presented as the mean $\pm \operatorname{SEM}(n=7)$. ${ }^{*} \mathrm{P}<0.05$ vs. respective sham group; ${ }^{\prime} \mathrm{P}<0.05$ vs. respective NT/tFI group. SP, stratum pyramidale; SO, stratum oriens; SR, stratum radiatum; NT, normothermia; HT, hyperthermia; $\mathrm{tFI}$, transient forebrain ischemia; NeuN, neuronal nuclei.

dramatically decreased (about $27.6 \%$ of the NT/tFI group) from 2 days post-tFI (Fig. $2 \mathrm{Ae}, \mathrm{Be}$ and $\mathrm{C}$ ), showing that the numbers of the $\mathrm{NeuN}^{+}$neurons at 5 days after tFI was similar to that in the NT/tFI group (Fig. 2Af, Bf and C).

F-JB positive cells. F-JB-positive cells, which are dead cells, were not shown in the CA1 region of the NT/sham (Fig. 3Ac, Ba, C). In the NT/tFI group, F-JB-positive cells were not found at 2 days after tFI (Fig. 3Ab, Bb and C), but many F-JB-positive cells were shown in the SP at 5 days after $\mathrm{tFI}$ (Fig. 3Ac, Bc and C).

In the HT/sham group, also F-JB-positive cells were not shown in the CA1 region (Fig. 3Ad, Bd and C). However, In the HT/tFI group, many F-JB-positive cells were found in the SP at 2 days after $\mathrm{tFI}$ (Fig. 3Ae, Be and C). At 5 days after tFI in this group, the distribution pattern of F-JB-positive cells was similar to that at 2 days after tFI (Fig. 3Af, Bf and C).

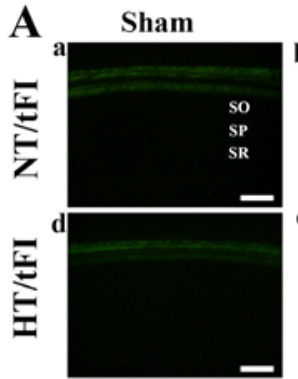

B
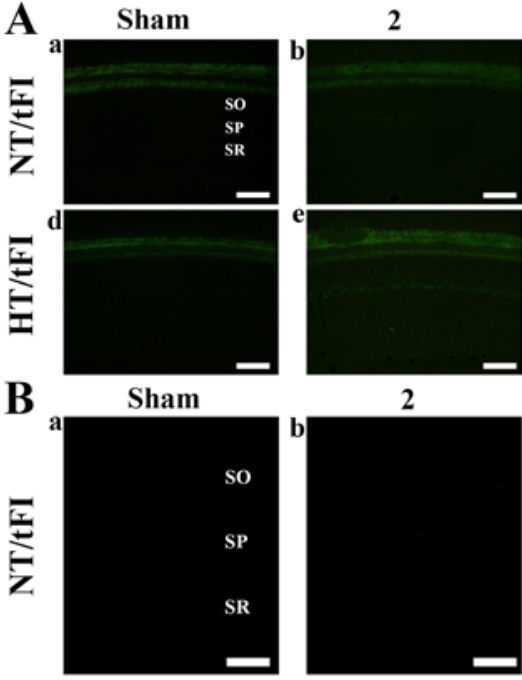

2
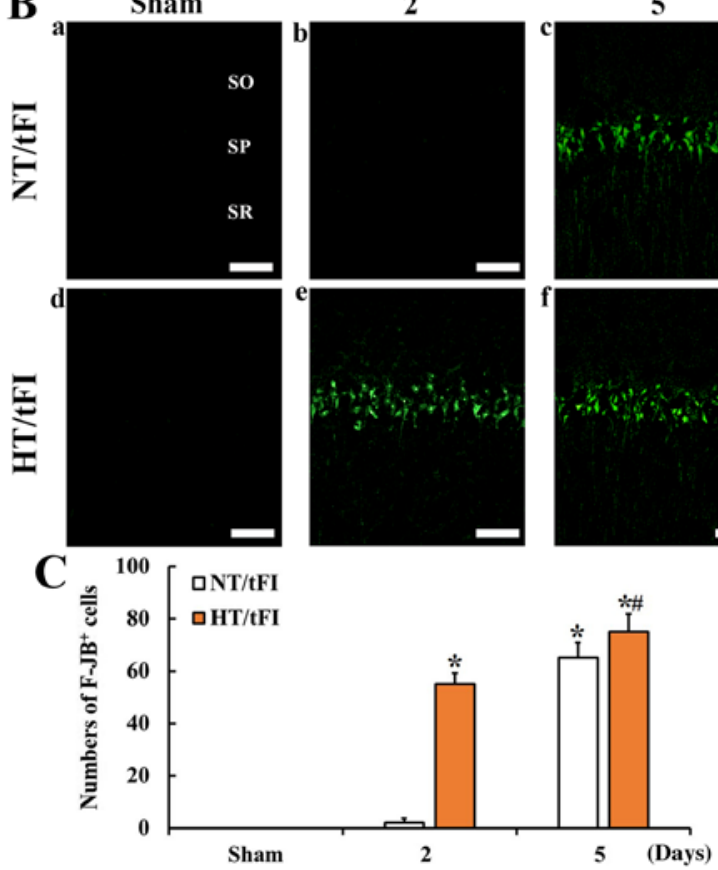

Figure 3. (A) Low (x10; scale bar, $200 \mu \mathrm{m}$ ) and (B) high (x40; scale bar, $50 \mu \mathrm{m}$ ) magnification of histofluorescence with F-JB in the CA1 region of the (a) NT/sham, (b and c) NT/tFI at 2 and 5 days after tFI, (d) HT/sham and (e and $\mathrm{f}$ ) HT/tFI groups at 2 and 5 days post-tFI. In the NT/tFI group, numerous $\mathrm{F}_{-} \mathrm{JB}^{+}$cells were detected in the SP 5 days after $\mathrm{tFI}$. However, in the $\mathrm{HT} / \mathrm{tFI}$ group, numerous $\mathrm{F}_{-} \mathrm{JB}^{+}$cells were observed from 2 days post-tFI. (C) Numbers of F-JB ${ }^{+}$cells in the CA1 region. The data are presented as the mean \pm SEM $(n=7)$. ${ }^{*} \mathrm{P}<0.05$ vs. respective sham group; ${ }^{\#} \mathrm{P}<0.05$ vs. respective NT/tFI group. SP, stratum pyramidale; SO, stratum oriens; SR, stratum radiatum; NT, normothermia; $\mathrm{HT}$, hyperthermia; $\mathrm{tFI}$, transient forebrain ischemia; F-JB, Fluoro-Jade B.

NMDAR1 protein level. NMDAR1 level in the CA1 region of the NT/tFI group was changed after tFI, showing that the level began to increase (111.3\%) at 1 day, was highest (121.7\%) at 2 days and very low (30.4\%) at 5 days after tFI when compared with that in the NT/sham group (Fig. 4). NMDAR1 level in the HT/sham group was similar to that in the NT/sham group (Fig. 4). However, in the HT/tFI group, NMDAR1 level was differently altered after tFI compared to the HT/tFI group, showing that the level was higher (113.1\%) at $6 \mathrm{~h}$, similar at 1 day, very lower (17.4\%) 2 days and low (13.2\%) at 5 days after tFI compared with that in the NT/tFI group (Fig. 4).

NMDAR1 immunoreactivity. NMDAR1 immunoreactivity in the NT/sham group was shown in the neuropil in the stratum oriens (SO) and radiatum (SR), not in the SP of the $\mathrm{CA} 1$ region (Fig. 5Aa and $\mathrm{Ba}$ ). In the $\mathrm{NT} / \mathrm{tFI}$ group, NMDAR1 immunoreactivity at $6 \mathrm{~h}$ post-tFI was similar to that in the NT/sham group (Fig. 5Ab and $\mathrm{Bb}$ ), but strong 


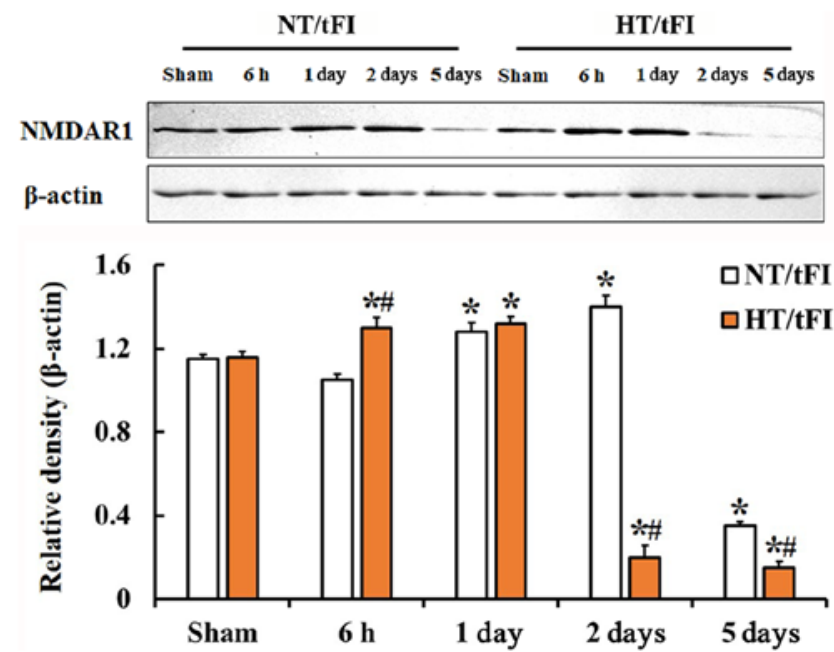

Figure 4. Western blot analyses of NMDAR 1 in the CA1 region of the $\mathrm{NT} / \mathrm{sham}, \mathrm{NT} / \mathrm{tFI}, \mathrm{HT} / \mathrm{sham}$ and HT/tFI groups at $6 \mathrm{~h}, 1$ day, 2 days and 5 days after tFI. The change in NMDAR1 expression in the NT/tFI group was different from that in the HT/tFI group. The graph indicates the relative optical density of the immunoblot bands. The data are presented as the mean \pm SEM ( $n=7)$. ${ }^{*}<<0.05$ vs. respective sham group; ${ }^{*} \mathrm{P}<0.05$ vs. respective $\mathrm{NT} / \mathrm{tFI}$ group. NT, normothermia; HT, hyperthermia; tFI, transient forebrain ischemia; NMDAR1, $N$-methyl-D-aspartate receptor 1.

NMDAR1 immunoreactivity was shown in cells in the SP and processes (dendrites) in the SR (Fig. 5Ab, Ac, Bb, Bc and C). At 2 days post-tFI, NMDAR1 immunoreactivity in the cells of the SP was increased compared with that at 1 day post-tFI, showing that NMDAR1-immunoreactive processes were broken (Fig. 5Ad, Bd and C). At 5 days post-tFI, NMDAR1-immunoreactive cells and fibers were decreased compared with those at 2 days post-tFI (Fig. 5Ae, Be and C).

In the HT/sham group, there was no significant difference in NMDAR1 immunoreactivity compared to that in the NT/sham group (Fig. 5Af, Bf and $\mathrm{C}$ ). In the HT/tFI group, NMDAR 1 immunoreactivity at $6 \mathrm{~h}$ was significantly higher $(143.9 \%)$ than that in the NT/tFI group, showing that its distribution pattern was similar to that at 1 day in the NT/tFI group (Fig. 5Ag, Bg and C). At 1 day post-tFI, numerous cells in the SP showed strong NMDAR1 immunoreactivity, thereafter, NMDAR1-immunoreactive structures were gradually decreased with time after tFI, showing that the ROD of NMDAR1 immunoreactivity was lower than that in the NT/tFI group (Fig. 5Ah, Ai, Aj, Bh, Bi, Bj and C).

\section{Discussion}

It has been reported that artificially elevated body temperature is closely associated with deterioration of neurological deficits, ischemic lesion (infarct), microvascular injury, and neuronal damage in various rodent models of brain ischemic insults (14,30-33). In addition, clinical data have shown that hyperthermia can increase infarction and worsen the outcome of ischemic injury in ischemic stroke patients $(20,21,34)$. Namely, hyperthermic condition during ischemic insults can markedly augment mortality and severity of ischemic damage than under a normothermic condition.

Our current study showed that hyperthermic preconditioning prior to $\mathrm{tFI}$ in the $\mathrm{HT} / \mathrm{tFI}$ group could significantly reduce short-term memory function evaluated by PAT compared to the control (NT/tFI group). This finding agrees with previous studies showing that cognitive impairment is accelerated after brain injury under hyperthermia $(35,36)$.

It has been reported that death of pyramidal cells in the gerbil hippocampal CA1 region following tFI under normothermic condition occurs at 4-5 days after tFI, and this phenomenon is known as 'delayed neuronal death' (1). However, in the HT/tFI group with memory deterioration, death (loss) of pyramidal neurons in the CA1 region was accelerated and deteriorated than that in the NT/tFI group. In particular, neuronal loss in the HT/tFI group began at 2 days post-tFI, while neuronal loss in the NT/tFI group was found at 5 days post-tFI. Although the present study did not confirm neuronal death at $6 \mathrm{~h}$ and 1day after tFI, this finding is similar to our previously published results showing that, under hyperthermic conditions before and during ischemia-reperfusion (IR), neuronal death began at 1 day post-tFI and was accelerated at 2-3 days post-tFI, and that hyperthermia increased the extent and severity of IR-induced neuronal death and glial activation in the hippocampal CA1 region $(13,23)$. Similar findings have been reported in a canine model of transient forebrain ischemia (37). Taken together, these results suggest that hyperthermic conditioning in ischemia can accelerate the extent and severity of neuronal death in ischemic brains.

NMDAR1 is one of $\mathrm{Ca}^{2+}$-permeable receptors that mediate glutamate excitotoxicity (38). In 1992, Choi reported that ischemia-induced excessive glutamate release might result in a pathological level of $\mathrm{Ca}^{2+}$ influx through NMDAR, leading to subsequent neuronal death (39). It has been reported that excessive glutamate release after ischemic brain injury is accentuated by hyperthermia in animal models of transient brain ischemia (40). Glutamate excitotoxicity is involved in the vulnerability of neurons following transient ischemia (41). It has been suggested that neuroprotection is provided by late NMDA receptor blockade (i.e., blocking of presynaptic release of glutamate after excessive activation of glutamate receptors and/or blocking of postsynaptic sensitization of NMDA receptors) (41-43). In our current study, the expression of NMDAR1 in pyramidal neurons of the CA1 region in the NT/tFI group was diversely altered after tFI. This finding is related to the process of damage and death of pyramidal neurons for 4-5 days after $\mathrm{tFI}$ under normothermic condition.

In this study, NMDAR1 expression in the HT/tFI group was early augmented in the pyramidal neurons and differently altered than that in the NT/tFI group. Castillo et al (44) have reported a glutamate-related relationship between increased body temperature and deterioration of symptoms in ischemic stroke patients clinically. In hyperthermic rats, significantly increased glutamate release has been observed in the brain following global ischemia (45). Therefore, the effect of hyperthermic condition on glutamate release might be mediated by overexpression of NMDARs under a hyperthermic condition. Results of our current study and previous studies suggest that accumulation of NMDAR1 in the early phase after tFI under hyperthermia plays a critical role in the deterioration of neuronal function after $\mathrm{tFI}$.

In conclusion, NMDAR1 expression in the HT/tFI group was earlier and more increased in pyramidal neurons located 


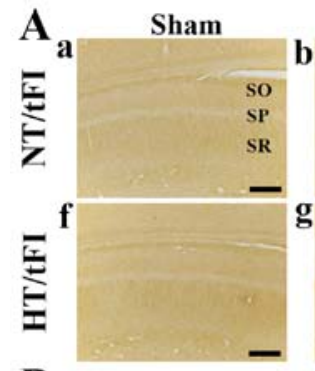

B
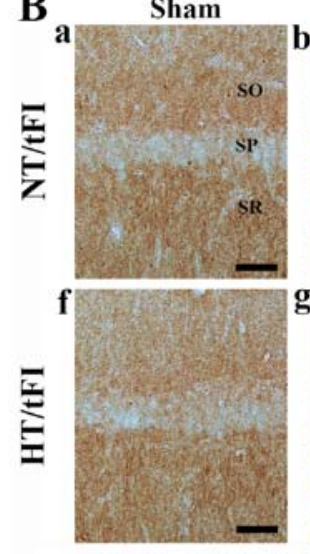

C
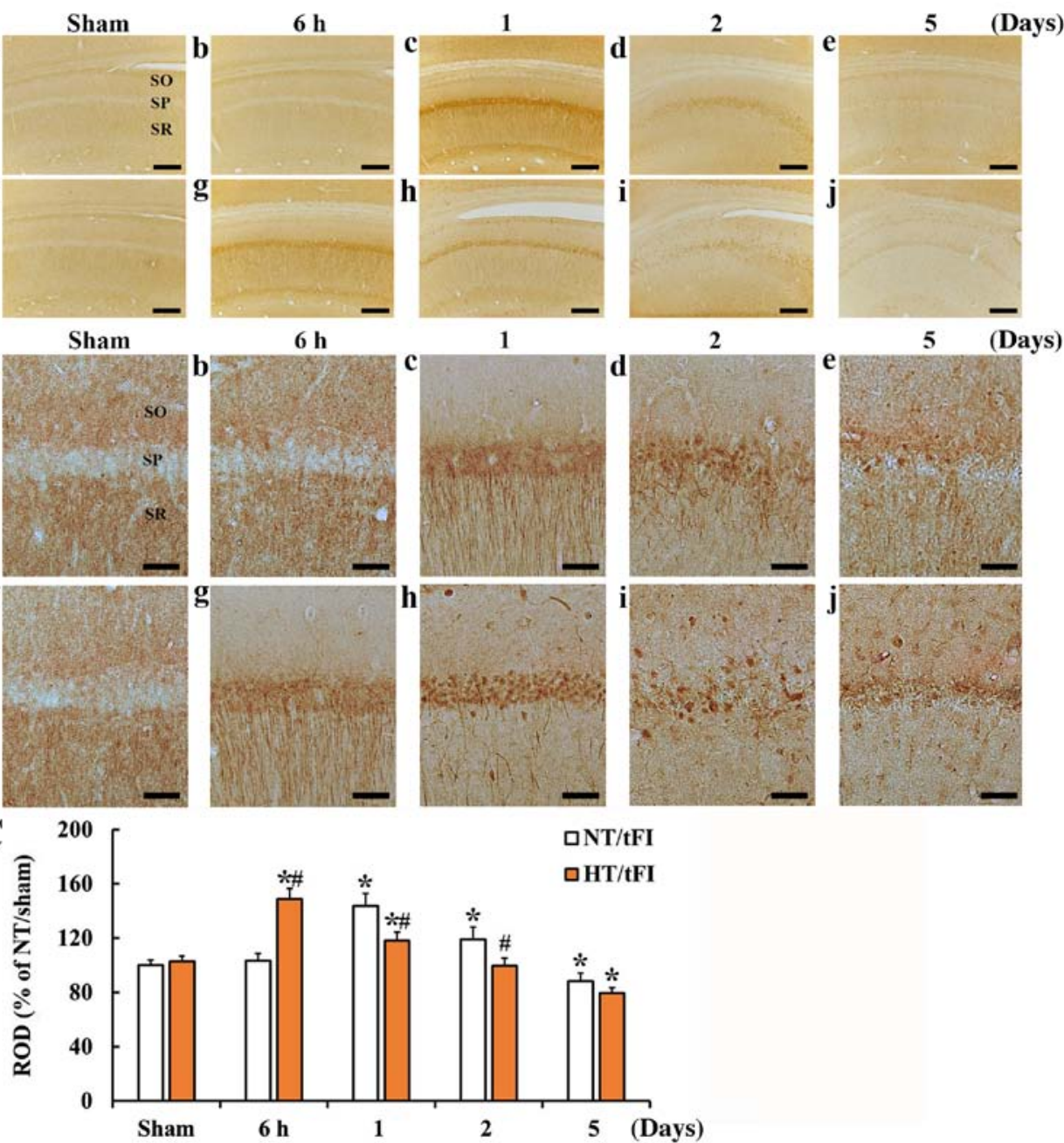

$\square \mathrm{NT} / \mathrm{tFI}$

$\square \mathrm{HT} / \mathrm{tFI}$

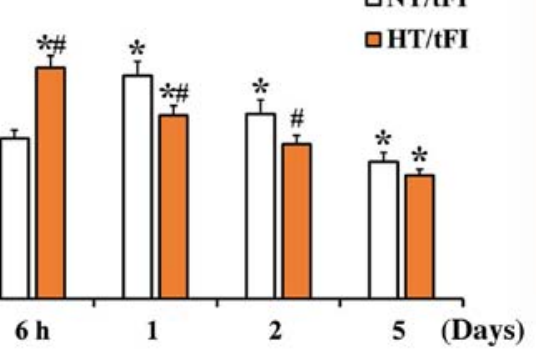

Figure 5. (A) Low (x10; scale bar, $200 \mu \mathrm{m}$ ) and (B) high (x40; scale bar, $50 \mu \mathrm{m}$ ) magnification of immunohistochemical staining for NMDAR1 in the CA1 region of the (a) NT/sham, (b-e) NT/tFI at 6 h, 1 day, 2 days and 5 days after tFI, (f) HT/sham and (g-j) HT/tFI groups at 6 h, 1 day, 2 days and 5 days after tFI. NMDAR1 immunoreactivity was hardly observed in the SP of the sham groups. In the NT/tFI group, strong NMDAR1 immunoreactivity was observed in the somata and processes at 1 and 2 days post-tFI. In the HT/tFI group, NMDAR 1 immunoreactivity was increased from $6 \mathrm{~h}$ post-tFI, indicating that the change pattern of NMDAR1 immunoreactivity was different from that in the NT/tFI group. (C) ROD of NMDAR1 immunoreactivity in the CA1 region. The data are presented as the mean \pm SEM $(n=7)$. ${ }^{P}<0.05$ vs. respective sham group; ${ }^{*} \mathrm{P}<0.05$ vs. respective $\mathrm{NT} / \mathrm{tFI}$ group. SP, stratum pyramidale; SO, stratum oriens; SR, stratum radiatum; NT, normothermia; HT, hyperthermia; tFI, transient forebrain ischemia; ROD, relative optical density; NMDAR1, $N$-methyl-D-aspartate receptor 1 .

in the CA1 stratum pyramidale than that in the NT/tFI group, suggesting that hyperthermia-mediated overexpression of NMDAR1 might be tightly associated with deterioration in memory decline and neuronal damage or death following transient ischemic insults.

\section{Acknowledgements}

Not applicable.

\section{Funding}

The present study was supported by the Cooperative Research Program for Agriculture Science and Technology Development (project no. PJ01329401) Rural Development Administration (Republic of Korea) and by the Bio-Synergy Research Project (grant no. NRF-2018M3A9C4076478) of the Ministry of Science and Information and Communication Technologies through the National Research Foundation.

\section{Availability of data and materials}

All data generated or analyzed during this study are included in this published article.

\section{Authors' contributions}

BK, JHA, JCL and MHW were responsible for experimental design, data analysis and drafting and reviewing the manuscript. DWK, TKL and YSK performed the experiments and data collection. MCS, JHC, YMK, JHP and IJK performed the data analysis and provided critical comments on the whole process of this work. All authors read and approved the final manuscript.

\section{Ethics approval and consent to participate}

The protocol of the present study was approved (approval no. KW-200113-1) on 13th January, 2020, by the 
Institutional Animal Care and Use Committee of Kangwon National University (Chuncheon, Republic of Korea).

\section{Patient consent for publication}

Not applicable.

\section{Competing interests}

The authors declare that they have no competing interests.

\section{References}

1. Kirino T: Delayed neuronal death in the gerbil hippocampus following ischemia. Brain Res 239: 57-69, 1982.

2. Kirino T, Tamura A and Sano K: Delayed neuronal death in the rat hippocampus following transient forebrain ischemia. Acta Neuropathol 64: 139-147, 1984

3. Chen J, Nagayama T, Jin K, Stetler RA, Zhu RL, Graham SH and Simon RP: Induction of caspase-3-like protease may mediate delayed neuronal death in the hippocampus after transient cerebral ischemia. J Neurosci 18: 4914-4928, 1998.

4. Benveniste H, Drejer J, Schousboe A and Diemer NH: Elevation of the extracellular concentrations of glutamate and aspartate in rat hippocampus during transient cerebral ischemia monitored by intracerebral microdialysis. J Neurochem 43: 1369-1374, 1984.

5. Arundine $M$ and Tymianski $M$ : Molecular mechanisms of calcium-dependent neurodegeneration in excitotoxicity. Cell Calcium 34: 325-337, 2003.

6. Kristian T and Siesjo BK: Calcium in ischemic cell death. Stroke 29: 705-718, 1998.

7. Young AB, Greenamyre JT, Hollingsworth Z, Albin R, D'Amato C, Shoulson I and Penney JB: NMDA receptor losses in putamen from patients with Huntington's disease. Science 241: 981-983, 1988.

8. Stanika RI, Winters CA, Pivovarova NB and Andrews SB Differential NMDA receptor-dependent calcium loading and mitochondrial dysfunction in CA1 vs. CA3 hippocampal neurons. Neurobiol Dis 37: 403-411, 2010.

9. Gao S, Yu Y, Ma ZY, Sun H, Zhang YL, Wang XT, Wang C, Fan WM, Zheng QY and Ma CL: NMDAR-mediated hippocampal neuronal death is exacerbated by activities of ASICla Neurotox Res 28: 122-137, 2015.

10. Seo JY, Yan BC, Park JH, Ahn JH, Kim IH, Lee JC, Kwon YG, Kim YM, Cho JH and Won MH: Comparison of the immunoreactivities of NMDA receptors between the young and adult hippocampal CA1 region induced by experimentally transient cerebral ischemia. J Neurol Sci 325: 108-114, 2013.

11. Benquet P, Gee CE and Gerber U: Transient brain ischemia: NMDA receptor modulation and delayed neuronal death. Med Sci (Paris) 24: 185-190, 2008 (In French).

12. Yanli L, Xizhou Z, Yan W, Bo Z, Yunhong Z, Zicheng L, Lingling $\mathrm{Y}$, Lingling $\mathrm{Y}$, Zhangao $\mathrm{C}$, Min $\mathrm{Z}$ and Zhi $\mathrm{H}$ : Clonidine preconditioning alleviated focal cerebral ischemic insult in rats via up-regulating p-NMDAR1 and down-regulating NMDAR2A/p-NMDAR2B. Eur J Pharmacol 793: 89-94, 2016.

13. Kim MJ, Cho JH, Cho JH, Park JH, Ahn JH, Tae HJ, Cho GS Yan BC, Hwang IK, Lee CH, et al: Impact of hyperthermia before and during ischemia-reperfusion on neuronal damage and gliosis in the gerbil hippocampus induced by transient cerebral ischemia. J Neurol Sci 348: 101-110, 2015.

14. Busto R, Dietrich WD, Globus MY and Ginsberg MD: The importance of brain temperature in cerebral ischemic injury. Stroke 20: 1113-1114, 1989.

15. Hsu SF, Niu KC, Lin CL and Lin MT: Brain cooling causes attenuation of cerebral oxidative stress, systemic inflammation, activated coagulation, and tissue ischemia/injury during heatstroke. Shock 26: 210-220, 2006

16. Dietrich WD, Busto R, Valdes I and Loor Y: Effects of normothermic versus mild hyperthermic forebrain ischemia in rats Stroke 21: 1318-1325, 1990.

17. Baena RC, Busto R, Dietrich WD, Globus MY and Ginsberg MD Hyperthermia delayed by 24 hours aggravates neuronal damage in rat hippocampus following global ischemia. Neurology 48: $768-773,1997$.
18. Morikawa E, Ginsberg MD, Dietrich WD, Duncan RC, Kraydieh S, Globus MY and Busto R: The significance of brain temperature in focal cerebral ischemia: Histopathological consequences of middle cerebral artery occlusion in the rat. J Cereb Blood Flow Metab 12: 380-389, 1992.

19. de Jonge JC, Wallet J and van der Worp HB: Fever worsens outcomes in animal models of ischaemic stroke: A systematic review and meta-analysis. Eur Stroke J 4: 29-38, 2019.

20. Seo WK, Yu SW, Kim JH, Park KW and Koh SB: The impact of hyperthermia and infection on acute ischemic stroke patients in the intensive care unit. Neurocrit Care 9: 183-188, 2008.

21. Wang Y, Lim LL, Levi C, Heller RF and Fisher J: Influence of admission body temperature on stroke mortality. Stroke 31: 404-409, 2000

22. Kim DW, Cho JH, Cho GS, Kim IH, Park JH, Ahn JH, Chen BH, Shin BN, Tae HJ, Hong S, et al: Hyperthermic preconditioning severely accelerates neuronal damage in the gerbil ischemic hippocampal dentate gyrus via decreasing SODs expressions. J Neurol Sci 358: 266-275, 2015.

23. Lee JC, Cho JH, Lee TK, Kim IH, Won MH, Cho GS, Shin BN, Hwang IK, Park JH, Ahn JH, et al: Effect of hyperthermia on calbindin-D 28k immunoreactivity in the hippocampal formation following transient global cerebral ischemia in gerbils. Neural Regen Res 12: 1458-1464, 2017.

24. Chen BH, Park JH, Lee YL, Kang IJ, Kim DW, Hwang IK, Lee CH, Yan BC, Kim YM, Lee TK, et al: Melatonin improves vascular cognitive impairment induced by ischemic stroke by remyelination via activation of ERK1/2 signaling and restoration of glutamatergic synapses in the gerbil hippocampus. Biomed Pharmacother 108: 687-697, 2018.

25. Lee JC, Park JH, Ahn JH, Kim IH, Cho JH, Choi JH, Yoo KY, Lee $\mathrm{CH}$, Hwang IK, Cho JH, et al: New GABAergic neurogenesis in the hippocampal CA1 region of a gerbil model of long-term survival after transient cerebral ischemic injury. Brain Pathol 26: 581-592, 2016.

26. Lee JC, Kim IH, Park JH, Ahn JH, Cho JH, Cho GS, Tae HJ, Chen $\mathrm{BH}$, Yan BC, Yoo KY, et al: Ischemic preconditioning protects hippocampal pyramidal neurons from transient ischemic injury via the attenuation of oxidative damage through upregulating heme oxygenase-1. Free Radic Biol Med 79: 78-90, 2015.

27. Radtke-Schuller S, Schuller G, Angenstein F, Grosser OS, Goldschmidt $\mathrm{J}$ and Budinger E: Brain atlas of the Mongolian gerbil (Meriones unguiculatus) in CT/MRI-aided stereotaxic coordinates. Brain Struct Funct 221 (Suppl 1): S1-S272, 2016.

28. Schmued LC and Hopkins KJ: Fluoro-Jade B: A high affinity fluorescent marker for the localization of neuronal degeneration. Brain Res 874: 123-130, 2000.

29. Sugawara T, Lewen A, Noshita N, Gasche Y and Chan PH: Effects of global ischemia duration on neuronal, astroglial, oligodendroglial, and microglial reactions in the vulnerable hippocampal CA1 subregion in rats. J Neurotrauma 19: 85-98, 2002.

30. Corbett D and Thornhill J: Temperature modulation (hypothermic and hyperthermic conditions) and its influence on histological and behavioral outcomes following cerebral ischemia. Brain Pathol 10: 145-152, 2000.

31. Dietrich WD, Halley M, Valdes I and Busto R: Interrelationships between increased vascular permeability and acute neuronal damage following temperature-controlled brain ischemia in rats. Acta Neuropathol 81: 615-625, 1991.

32. Barber PA, Hoyte L, Colbourne F and Buchan AM: Temperature-regulated model of focal ischemia in the mouse: A study with histopathological and behavioral outcomes. Stroke 35 : 1720-1725, 2004.

33. Campos F, Blanco M, Barral D, Agulla J, Ramos-Cabrer P and Castillo J: Influence of temperature on ischemic brain: Basic and clinical principles. Neurochem Int 60: 495-505, 2012.

34. Reith J, Jørgensen HS, Pedersen PM, Nakayama H, Raaschou HO, Jeppesen LL and Olsen TS: Body temperature in acute stroke: Relation to stroke severity, infarct size, mortality, and outcome. Lancet 347: 422-425, 1996.

35. Titus DJ, Furones C, Atkins CM and Dietrich WD: Emergence of cognitive deficits after mild traumatic brain injury due to hyperthermia. Exp Neurol 263: 254-262, 2015.

36. Walter EJ and Carraretto M: The neurological and cognitive consequences of hyperthermia. Crit Care 20: 199, 2016. 
37. Wass CT, Lanier WL, Hofer RE, Scheithauer BW and Andrews AG: Temperature changes of $>$ or $=1$ degree $\mathrm{C}$ alter functional neurologic outcome and histopathology in a canine model of complete cerebral ischemia. Anesthesiology 83: 325-335, 1995.

38. Moriyoshi K, Masu M, Ishii T, Shigemoto R, Mizuno N and Nakanishi S: Molecular cloning and characterization of the rat NMDA receptor. Nature 354: 31-37, 1991.

39. Choi DW: Excitotoxic cell death. J Neurobiol 23: 1261-1276, 1992.

40. Ginsberg MD and Busto R: Combating hyperthermia in acute stroke: A significant clinical concern. Stroke 29: 529-534, 1998.

41. Nishizawa Y: Glutamate release and neuronal damage in ischemia. Life Sci 69: 369-381, 2001.

42. Traystman RJ: Animal models of focal and global cerebral ischemia. ILAR J 44: 85-95, 2003.
43. Dhawan J, Benveniste H, Luo Z, Nawrocky M, Smith SD and Biegon A: A new look at glutamate and ischemia: NMDA agonist improves long-term functional outcome in a rat model of stroke. Future Neurol 6: 823-834, 2011.

44. Castillo J, Davalos A, Marrugat J and Noya M: Timing for fever-related brain damage in acute ischemic stroke. Stroke 29: 2455-2460, 1998.

45. Campos F, Pérez-Mato M, Agulla J, Blanco M, Barral D, Almeida A, Brea D, Waeber C, Castillo J and Ramos-Cabrer P: Glutamate excitoxicity is the key molecular mechanism which is influenced by body temperature during the acute phase of brain stroke. PLoS One 7: e44191, 2012.

c) (i) (2) This work is licensed under a Creative Commons Attribution-NonCommercial-NoDerivatives 4.0 International (CC BY-NC-ND 4.0) License. 